\title{
Alluvione di Messina 2009: NGAL in due pazienti con Crush Syndrome
}

\section{Donato ${ }^{1}$, A. Noto ${ }^{2}$, A. Lacquaniti ${ }^{1}$, A. Versaci ${ }^{2}$, M. Giardina ${ }^{2}$, D. Bolignano ${ }^{1}$, F. Spinelli ${ }^{3}$, A. David ${ }^{2}$, M. Buemi ${ }^{1}$}

${ }^{1}$ Cattedra di Nefrologia, Dipartimento di Medicina Interna, Università di Messina, Messina

${ }^{2}$ Cattedra di Anestesia e Rianimazione Cardio-Vascolare e Toracica, Università di Messina, Messina

${ }^{3}$ Cattedra di Chirurgia Vascolare, Università di Messina, Messina

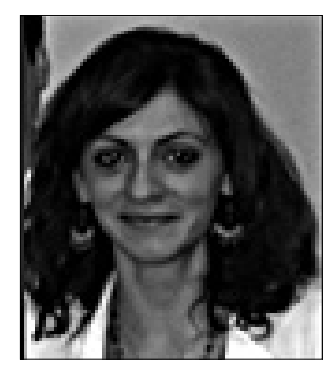

Valentina Donato

\section{Introduzione}

La Crush Syndrome o Sindrome da schiacciamento è una condizione clinica particolarmente severa caratterizzata da ischemia/riperfusione che si instaura in seguito alla compressione lenta e prolungata di uno o più arti e al consequenziale interessamento del sistema cardiovascolare e renale. Tende a verificarsi sempre più sovente in seguito a eventi ambientali catastrofici, quali terremoti, valanghe e alluvioni e rappresenta la seconda causa più frequente di mortalità disastro-correlata costituendo di per sé un problema organizzativo rilevante dal punto di vista della gestione da parte delle strutture sanitarie $(1,2)$.

La storia della Crush Syndrome non è nuova dalle nostre parti, essendo già stata descritta nel 1908 dal tedesco Von Colmers che la definì come sindrome caratterizzata da grave danno muscolare e insufficienza renale secondaria all'intrappolamento dei corpi sotto le macerie del terremoto che coinvolse Messina (3).

\section{Caso clinico}

La recente alluvione che ha colpito i centri periferici della città di Messina ha provocato la morte di circa 50 persone ed oltre 300 feriti.

Descriviamo i casi clinici giunti alla nostra osservazione. Due donne, rispettivamente di 28 (P.C.) e 46 anni (A.A.), entrambe senza anamnesi remota che documentasse alcuna patologia rilevante.

P.C. rimasta per 14 ore sotto le macerie in seguito al crollo di un palazzo e A.A. rimasta 12 ore bloccata nel fango prima di essere recuperate e ricoverate presso l'Unità di Terapia Intensiva. Sebbene le due pazienti fos- sero state colpite da un identico evento compressivo, con sindrome compartimentale agli arti inferiori e anuria, presentavano parametri ematochimici differenti.

Esami di Laboratorio all'ingresso della paziente P.C.

Temperatura corporea: $37^{\circ} \mathrm{C}$.

PA: 100/60 mmHg; FC: 89 bpm; Respiratory rate: 12 bpm; $\mathrm{FIO}_{2}: 100 \%$;

pH: 7,144,; $\mathrm{pCO}_{2}$ : 45,7 mmHg; $\mathrm{pO}_{2}: 364 \mathrm{mmHg}, \mathrm{cHCO}_{3}$ : 15,3 mmol/L; BE: -13,1 mmol/L; Osm: $278 \mathrm{mOsm} / \mathrm{kg}$; $\mathrm{O}_{2} \mathrm{Hb}: 97,5 \%$; $\mathrm{SO}_{2}$ : 99,8\%;

Creatininemia: $1,3 \mathrm{mg} / \mathrm{dL} ; \mathrm{K}^{+}: 6,36 \mathrm{mmol} / \mathrm{L} ; \mathrm{Na}^{+}: 137,8$ $\mathrm{mmol} / \mathrm{L} ; \mathrm{Cl}: 103 \mathrm{mmol} / \mathrm{L} ;$ Glicemia: $133 \mathrm{mg} / \mathrm{dL} ; \mathrm{Bi}-$ lirubina: 0,6 mg/dL; CPK: $90286 \mathrm{U} / \mathrm{L}$; Mioglobina: $>4000$ mg/L; LDH: 3876.

Score APACHE: 24; Score SOFA: 10.

WBC: $20.000 \mathrm{~mm}^{3}$; Hb: 7,1 g/dL; PLT: $150.000 \mathrm{~mm}^{3}$. sNGAL: $571 \mathrm{ng} / \mathrm{mL}$.

Essendo sotto ventilazione automatica, seguiva intervento di rivascolarizzazione alla gamba sinistra ed iniziava terapia dialitica con metodica diffusivo-convettiva: Continuous Veno-Venus Hemodiafiltration (CVVHDF) intraoperatoria.

\section{Esami ematochimici all'uscita della sala operatoria}

pH: 7,495; $\mathrm{pCO}_{2}: 33,5 \mathrm{mmHg} ; \mathrm{cHCO}_{3}: 15,3 \mathrm{mmol} / \mathrm{L} ; \mathrm{BE}$ : $2 \mathrm{mmol} / \mathrm{L}$; Osm: $270 \mathrm{mOsm} / \mathrm{kg}$;

$\mathrm{pO}_{2}$ : 250,5 mmHg; $\mathrm{O}_{2} \mathrm{Hb}: 97,8 \% ; \mathrm{SO}_{2}: 99,6 \% ; \mathrm{FIO}_{2}:$ 0,50\%; $\mathrm{K}^{+}: 5,62 \mathrm{mmol} / \mathrm{L} ; \mathrm{Na}^{+}: 133.5 \mathrm{mmol} / \mathrm{L} ; \mathrm{Cl}^{-}: 97,4 \mathrm{mmol} / \mathrm{L}$; Hb: 7,2 g/dL; Hct: 22,4\%, Glicemia: $148 \mathrm{mg} / \mathrm{dL}$.

Parametri ematochimici della paziente A.A. al momento del ricovero

Temperatura corporea: $36,6^{\circ} \mathrm{C}$. 
PA:110/60 mmHg; FC: 70 bpm; Respiratory rate: 18 bpm, $\mathrm{FIO}_{2}: 30 \%$,

pH: 7,316; pCO2: 36,2 mmHg; pO2: 71,8 mmHg; cHCO3: 18,1 mmol/L; BE: -7.4 mmol/L; Osm: $285 \mathrm{mOsm} /$ kg; O2Hb: 91,5\%; SO2: 93,2\%;

Creatininemia: $1,2 \mathrm{mg} / \mathrm{dL}$; $+:$ 4,95 mmol/L; Na+: 140,8 mmol/L; Cl-: 100,5 mmol/L; Glicemia: 163 mg/dL; Bilirubina: 0,3 mg/dL; CPK: $70257 \mathrm{U} / \mathrm{L}$; Mioglobina: > $4000 \mathrm{mg} / \mathrm{L}$; LDH: 2656.

Score APACHE: 18; Score SOFA: 9.

Hb: 12,3 g/dL; Hct: 35,3\%; WBC: $23.700 \mathrm{~mm}^{3}$; PLT: $360.000 \mathrm{~mm}^{3}$.

sNGAL: $176 \mathrm{ng} / \mathrm{mL}$.

Veniva posta per entrambe diagnosi di Crush Syndrome, rabdomiolisi, disturbi della coagulazione, sepsi e "Acute Kidney Injury" (AKI).

I valori di sNGAL, WBC,CPK, PLT venivano monitorizzati giornalmente (Fig. 1).

Entrambe le pazienti, essendo anuriche, venivano sottoposte a terapia dialitica continua $24 \mathrm{~h} / 24 \mathrm{~h}$ con metodica CVVHDF previo posizionamento di catetere centrale in vena giugulare destra.

A causa della comparsa di sindrome compartimentale ad entrambi gli arti, le due pazienti venivano sottoposte ad intervento chirurgico con creazione di fasciotomie degli arti inferiori.

Dalle prime colture eseguite, P.C. presentava ferite infette da Stenotrophomonas maltophilia, Acinetobacter baumannii e klebsiella. In ottava giornata si aveva inoltre la positività colturale per Escherichia

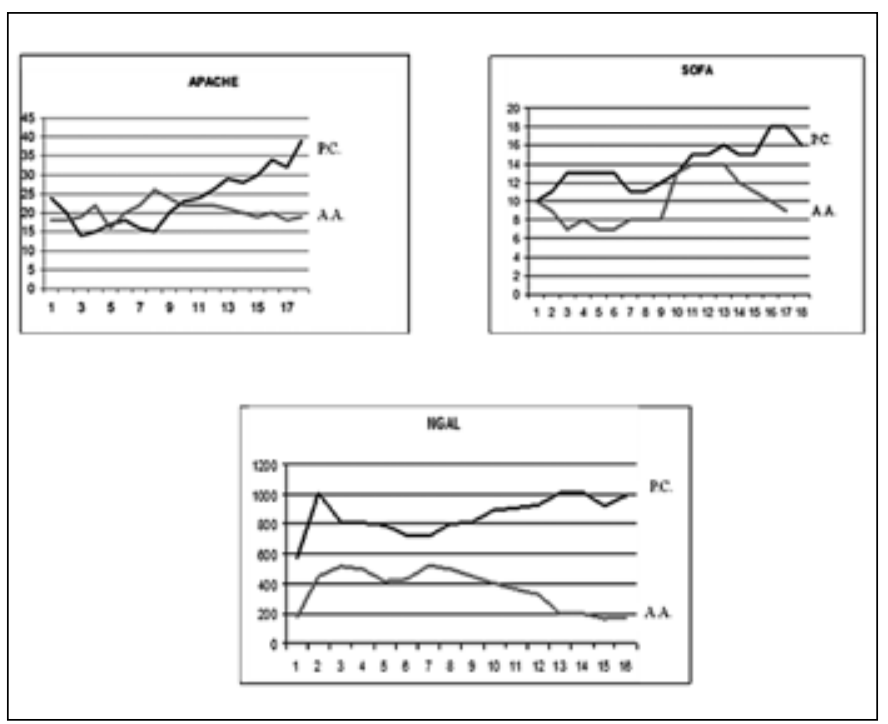

Fig. 1 - Andamento dell'NGAL e degli scores SOFA e APACHE nelle pazienti P.C. e A.A. nel corso dei giorni di degenza.
Coli ed Enterococcus fecalis. A.A. risultava positiva solo per Acinetobacter Baumanii.

Per tutto il periodo del ricovero si praticava quindi terapia antibiotica mirata.

Dopo nove giorni dall'ingresso in reparto, le pazienti venivano sottoposte a sedute giornaliere di camera iperbarica. In decima giornata A.A. riprendeva l'alimentazione enterale.

Al contrario, le condizioni di P.C. peggioravano progressivamente, persisteva l'anuria e i valori di NGAL rimanevano sempre elevati $(1000 \mathrm{ng} / \mathrm{mL})$.

A seguito di una grave crisi di Insufficienza Respiratoria Acuta, in $17^{\text {a }}$ giornata, sopraggiungeva la morte.

A.A. essendo stata sempre vigile, respirava spontaneamente, proseguiva sedute di camera iperbarica e terapia dialitica continua sino alla $23^{a}$ giornata. Con la ripresa della diuresi si riduceva la prescrizione dialitica fino alla sua completa sospensione che avveniva in $32^{\mathrm{a}}$ giornata.

I valori di NGAL diminuivano in maniera significativa dall' $8^{\mathrm{a}}$ giornata di ricovero per raggiungere valori ai limiti massimi della norma $(150 \mathrm{ng} / \mathrm{mL})$ alla dimissione: $49^{\circ}$ giorno.

\section{Discussione}

Le nostre due pazienti sono state vittime di una patologia multiorgano con Crush Syndrome, come conseguenza di eventi traumatici, instaurati a seguito di un'alluvione che ha colpito un piccolo centro periferico della città di Messina. A causa della compressione muscolare, i miociti sono stati danneggiati con rilascio di costituenti intracellulari nella circolazione sistemica. Ciò viene definito rabdomiolisi. Uno dei componenti chiave rilasciati è la mioglobina, carrier dell'ossigeno di 18000 Da simile all'emoglobina. A differenza dell'emoglobina contiene solo una metà di Eme. La mioglobina è filtrata dai glomeruli e raggiunge i tubuli dove provoca ostruzione, necrosi tubulare e deficit della funzione renale (Fig. 2). Altri componenti intracellulari come protoni, fosfato, nucleotidi, sono rilasciati dai muscoli danneggiati e contribuiscono alla fisiopatologia della sindrome da schiacciamento. La struttura cellulare degli arti inferiori delle nostre pazienti, a causa della compressione, è stata alterata dall'edema e dal rilascio di sostanze, quali chinine e istamina, che hanno provocato incremento di volume dello spazio interstiziale e accentuazione dello stato ischemico (4). Inoltre, la disidratazione e la sepsi hanno contribuito all'instaurazione dell'Insufficienza Renale Acuta oggi definita AKI (Acute Kidney Injury).

Per seguire l'evoluzione del quadro clinico e della funzione renale delle nostre due pazienti oltre ai classici "markers" quali creatinina, mioglobina, $\mathrm{CPK}, \mathrm{LDH}$, abbiamo valutato anche il comportamento del Neutrophil Gelatinase-Associated Lipocalin (NGAL), piccola proteina di $25 \mathrm{kDa}$ appar- 
tenente alla superfamiglia delle lipocaline, massivamente rilasciata dalle cellule tubulari dopo stimoli lesivi di varia natura (5).

Nei nostri due casi, al momento del ricovero, i livelli sierici di NGAL erano particolarmente elevati sia in P.C. (571 ng/ $\mathrm{mL})$ che in A.A. ( $176 \mathrm{ng} / \mathrm{mL})$ mentre la creatinina plasmatica rimaneva nel "range" della norma. Questi dati confermano il ruolo dell'NGAL come marker particolarmente precoce dellinsufficienza renale acuta, rispetto alla creatinina. La creatinina, come è noto, può variare significativamente con l'età, il sesso, la massa muscolare e lo stato di idratazione, può essere falsata da particolari trattamenti farmacologici, ma soprattutto può rimanere inalterata finché una consistente percentuale della funzione renale non diviene compromessa (6).

Inoltre i livelli sierici di NGAL nelle due pazienti suggeriscono una differente gravità del quadro clinico e si correlavano bene con gli score Apache e SOFA, ma l'aspetto più significativo del profilo di NGAL, durante tutto il periodo di ricovero, è stato l'evoluzione della funzione renale. Infatti I'NGAL sierico di P.C. partendo da valori significativamente alti si è incrementato ulteriormente al terzo giorno con prosecuzione della terapia dialitica, per raggiungere lo "zenit" prima della morte avvenuta alla $17^{\text {a }}$ giornata di ricovero.

Mentre NGAL in A.A., dall'ottavo giorno, si è progressivamente ridotto fino a raggiungere i livelli massimi di normalità alla ripresa funzionale del rene alla $23^{\text {a }}$ giornata. Pertanto, l'NGAL nei primi giorni di ricovero ha permesso di evidenziare nei nostri due casi, e in maniera abbastanza precoce, la gravità della situazione clinica generale oltre a definire il differente rischio per quanto riguarda l'evoluzione dell'insufficienza renale (7).

La Paziente P.C. presentava livelli ematici di NGAL significativamente elevati rispetto ad A.A., con un andamento a plateau che si concludeva con la morte dopo diciassette giorni. Questo comportamento potrebbe essere valutato come predittore dell'evento morte così come dimostrato in un nostro recente lavoro su 46 pazienti anziani affetti da cardiopatia. Le curve di sopravvivenza di Kaplan-Meier, nei soggetti con NGAL basale superiore a $783 \mathrm{ng} / \mathrm{mL}$ hanno mostrato una mortalità significativamente aumentata con Hazard Ratio di morte di 4,08 (8).

I casi clinici giunti alla nostra osservazione, pertanto, rappresentano due esempi paradigmatici del ruolo predittivo dell'NGAL. Come già precedenti studi hanno dimostrato, questa proteina ha permesso di diagnosticare precocemente l'AKI, mentre i differenti livelli sierici sono stati in grado di predire la diversa evoluzione delle capacità funzionali renali nonché la prognosi infausta per la paziente P.C.

Questa nostra esperienza vuole essere da stimolo all'uso in nefrologia del biomarker NGAL, che da semplice fattore antibatterico del sistema dell'immunità naturale, occupa

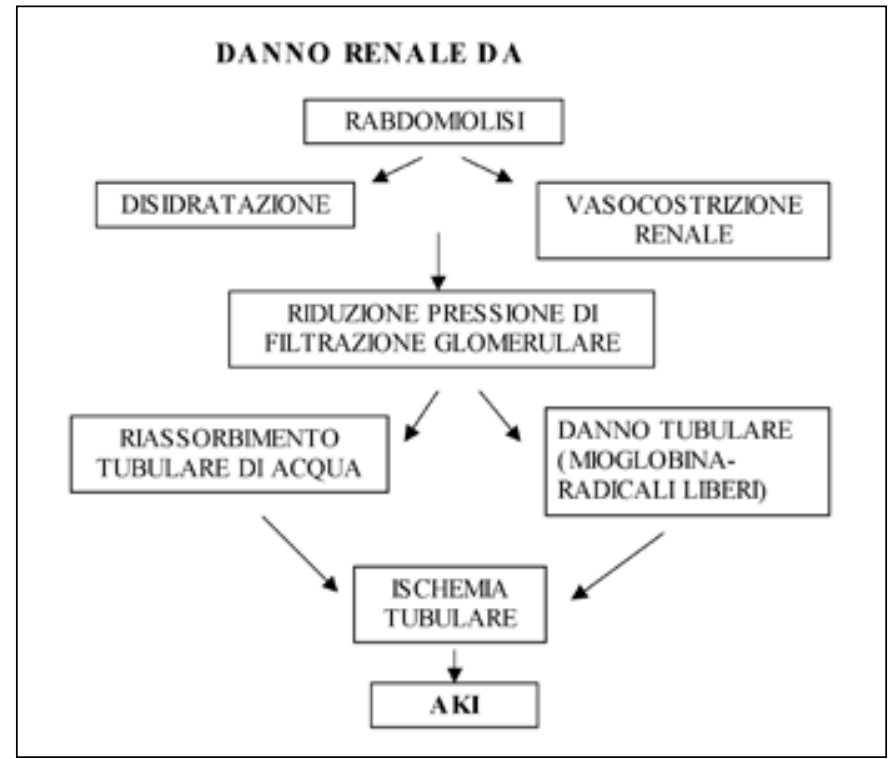

Fig. 2 - Meccanismi fisiopatologici dell'insufficienza renale acuta (AKI) da rabdomiolisi.

adesso un ruolo centrale nei processi fisiologici e patologici di quasi tutti gli apparati dell'organismo umano (9).

\section{Riassunto}

Neutrophil Gelatinase-Associated Lipocalin (NGAL) è uno dei più promettenti biomarcatori utilizzati nella diagnosi di "Acute Kidney Injury" (AKI), dal momento che il suo incremento è un buon predittore a breve termine dello sviluppo di insufficienza renale acuta in notevole anticipo rispetto all'incremento dei valori della creatinina sierica. Riportiamo la nostra esperienza di un caso di Crush Syndrome di due pazienti vittime dell'alluvione che ha coinvolto Messina. Lo sviluppo di AKI in seguito a Crush Syndrome è la seconda causa più comune di morte in seguito a terremoti o altri disastri naturali ma allo stesso tempo è una complicanza disastro-correlata che può essere reversibile in particolar modo in caso di diagnosi precoce e di altrettanto precoce trattamento. In questo caso, l'NGAL ci ha permesso di fare una diagnosi precoce di AKI preannunciando le alterazioni dei classici marker come la creatinina, inoltre abbiamo notato la correlazione diretta tra i valori di NGAL, l'evoluzione del danno renale e la prognosi per le due pazienti.

Parole chiave: Sindrome da sciacciamento, Neutrophil Gelatinase-Associated Lipocalin (NGAL), Danno renale acuto o Acute Kidney Injury (AKI) 
Indirizzo degli Autori:

Prof. Michele Buemi

Via salita Villa Contino 30

98100 Messina, Italy

buemim@unime.it

\section{TEST di VERIFICA - 1}

\section{1) Cos'è la rabdomiolisi?}

a) Una condizione clinica dovuta alla compressione prolungata del muscolo striato

b) Rilascio in circolo di sostanze dovute al danneggiamento dei miociti, quali potassio, mioglobina ed acido lattico

c) Precipitazione di mioglobina a livello tissutale

2) Danno renale acuto in Crush Syndrome: a cosa è dovuto?

a) Danno da ischemia/riperfusione

b) Precipitazione mioglobina al livello tubulare

c) Danneggiamento del glomerulo

d) Tutte le precedenti

e) $a+b$

\section{3) L'NGAL...}

a) Correla negativamente con lo sviluppo di AKI

b) Aumenta precocemente in caso di AKI rispetto alla creatinina

c) Il suo andamento correla con la gravità della funzione renale nonché con la prognosi

d) $\mathrm{B}+\mathrm{C}$

e) È fattore antibatterico del sistema dell'immunità naturale nonché marker di danno renale

f) $\mathrm{B}+\mathrm{c}+\mathrm{e}$

4) Le variazioni dei livelli di NGAL possono essere prese in considerazione per valutare la prognosi di una paziente?

a) Si perché più studi dimostrano come valori elevati di NGAL correlino con l'evento morte

b) No perché l'NGAL è un marcatore esclusivamente di danno renale cronico

c) Sì perché l'NGAL è un marcatore di danno renale acuto
5) La principale complicanza della sindrome da schiacciamento è:
a) L'AKI
b) La rabdomiolisi
c) L'ischemia e la riperfusione a valle dell'arto compresso
d) La sepsi
e) Nessuna delle precedenti
f) Tutte le precedenti

\section{6) Come viene diagnosticata attualmente l'AKI?}

a) Valutando l'incremento dei valori della creatinina plasmatica nel tempo

b) Monitorando la diuresi

c) Grazie al dosaggio di nuovi marcatori come l'NGAL

d) $a+b$

e) $a+c$

Le risposte corrette alle domande sono a pag. 25

\section{Bibliografia}

1. Sever MS, Vanholder R, Lameire N. Management of crushrelated injuries after disasters. New Engl J Med 2006; 354: 1052-63.

2. Sever MS, Lameire N, Vanholder R. Renal disaster relief: from theory to practice. Nephrol Dial Transplant 2009; 24: 1730-5.

3. Efstratiadis G, Voulgaridou A, Nikiforou D, et al. Rhabdomyolysis updated. Hyppokratia 2007; 11: 129-37.

4. Wei Q, Baihai S, Ping F, et al. Successful treatment of crush syndrome complicated with multiple organ dysfunction syndrome using hybrid continuous renal replacement therapy. Blood Purif 2009; 28: 175-80.

5. Flower DR. The lipocalin protein family: structure and function. Biochem J 1996; 318: 1-14.

6. Cruz DN, de Cal M, Garzotto F, et al. Plasma neutrophil gelatinase-associated lipocalin is an early biomarker for acute kidney injury in an adult ICU population. Intensive Care Med 2010; 36: 444-51.

7. Bolignano D, Lacquaniti A, Coppolino G, et al. Neutrophil gelatinase-associated lipocalin (NGAL) and progression of chronic kidney disease. Clin J Am Soc Nephrol 2009; 4: 337-44.

8. Bolignano D, Basile G, Parisi P, et al. Increased plasma neutrophil gelatinase-associated lipocalin levels predict mortality in elderly patients with chronic heart failure. Rejuvenation Res 2009; 12: 7-14.

9. Bolignano D, Buemi M. Neutrophil gelatinase-associated lipocalin (NGAL): un biomarker oltre i confini della nefrologia. Cosenza: EdiBios, 2009. 